\title{
Numerical Solution of a Kind of Fractional Parabolic Equations via Two Difference Schemes
}

\author{
Abdon Atangana ${ }^{1}$ and Dumitru Baleanu ${ }^{2,3,4}$ \\ ${ }^{1}$ Institute for Groundwater Studies, Faculty of Natural and Agricultural Sciences, University of the Free State, \\ Bloemfontein 9300, South Africa \\ ${ }^{2}$ Department of Chemical and Materials Engineering, Faculty of Engineering, King Abdulaziz University, P.O. Box 80204, \\ Jeddah 21589, Saudi Arabia \\ ${ }^{3}$ Department of Mathematics and Computer Sciences, Cankaya University, Faculty of Art and Sciences, Balgat, 06530 Ankara, Turkey \\ ${ }^{4}$ Institute of Space Sciences, P.O. Box MG-23, Magurele, 76900 Bucharest, Romania
}

Correspondence should be addressed to Abdon Atangana; abdonatangana@yahoo.fr

Received 5 August 2013; Accepted 23 August 2013

Academic Editor: Soheil Salahshour

Copyright (C) 2013 A. Atangana and D. Baleanu. This is an open access article distributed under the Creative Commons Attribution License, which permits unrestricted use, distribution, and reproduction in any medium, provided the original work is properly cited.

\begin{abstract}
A kind of parabolic equation was extended to the concept of fractional calculus. The resulting equation is, however, difficult to handle analytically. Therefore, we presented the numerical solution via the explicit and the implicit schemes. We presented together the stability and convergence of this time-fractional parabolic equation with two difference schemes. The explicit and the implicit schemes in this case are stable under some conditions.
\end{abstract}

\section{Introduction}

A parabolic partial differential equation is a type of secondorder partial differential equations (PDEs), describing a wide family of problems in science including heat diffusion and ocean acoustic propagation, in physical or mathematical systems with a time variable, which behave essentially like heat diffusing through a solid [1-4]. This mathematical model is a simplified description of physical reality expressed in mathematical terms. Thus, the investigation of the exact or approximate solution helps us to understand the means of these mathematical models. In most cases, it is difficult, or infeasible, to find the analytical solution or good numerical solution of the problems. Numerical solutions or approximate analytical solutions become necessary. Numerical methods typically yield approximate solutions to the governing equation through the discretization of space and time and can relax the rigid idealized conditions of analytical models or lumped-parameter models. They can, therefore, be more realistic and flexible for simulating field conditions. Within the discredited problem domain, the variable internal properties, boundaries, and stresses of the system are approximated. One of the most important aspects of this numerical method is the study of the stability and convergence of the numerical method $[5,6]$.

The purpose of this work is to study the stability and the convergence of the numerical scheme of the parabolic equation of the following form:

$$
\begin{gathered}
\partial_{t} v(x, t)+{ }_{0}^{C} D_{t}^{\alpha} v(x, t)-a(x, t) \partial_{x x}^{2} v(x, t)+\delta v(x, t) \\
=f(x, t), \quad v(0, x)=0, \\
0 \leq x \leq 1, \quad 0<\alpha \leq 1, \\
v(x, 0)=v(0, t), \quad v_{x}(t, 0)=v_{t}(x, 0),
\end{gathered}
$$

where $f(x, t)$ is sufficiently given smooth function and $a(x, t) \geq 0$. Here, $\delta$ is a sufficiently large positive constant.

\section{Useful Tools for the Fractional Calculus}

Definition 1 (see [7-17]). A real function $f(x), x>0$, is said to be in the space $C_{\mu}, \mu \in \mathbb{R}$, if there exists a real number $p>\mu$, such that $f(x)=x^{p} h(x)$, where $h(x) \in C[0, \infty)$, and it is said to be in space $C_{\mu}^{m}$ if $f^{(m)} \in C_{\mu}, m \in \mathbb{N}$. 
Definition 2 (see [7-16]). The Riemann-Liouville fractional integral operator of order $\alpha \geq 0$, of a function $f \in C_{\mu}, \mu \geq-1$, is defined as

$$
\begin{gathered}
J^{\alpha} f(x)=\frac{1}{\Gamma(\alpha)} \int_{0}^{x}(x-t)^{\alpha-1} f(t) d t, \quad \alpha>0, x>0, \\
J^{0} f(x)=f(x) .
\end{gathered}
$$

Properties of the operator can be found in [7-15]; we only mention the following:

$$
\begin{gathered}
\text { for } f \in C_{\mu}, \quad \mu \geq-1, \quad \alpha, \beta \geq 0, \quad \gamma>-1, \\
J^{\alpha} J^{\beta} f(x)=J^{\alpha+\beta} f(x), \quad J^{\alpha} J^{\beta} f(x)=J^{\beta} J^{\alpha} f(x), \\
J^{\alpha} x^{\gamma}=\frac{\Gamma(\gamma+1)}{\Gamma(\alpha+\gamma+1)} x^{\alpha+\gamma} .
\end{gathered}
$$

Definition 3. The Caputo fractional-order derivative is given as follows [7-10]:

$$
\begin{array}{r}
{ }_{0}^{C} D_{x}^{\alpha}(f(x))=\frac{1}{\Gamma(n-\alpha)} \int_{0}^{x}(x-t)^{n-\alpha-1} \frac{d^{n} f(t)}{d t^{n}} d t, \\
n-1 \leq \alpha \leq n .
\end{array}
$$

Definition 4. The Riemann-Liouville fractional-order derivative is given as follows [8-16]:

$$
\begin{array}{r}
D_{x}^{\alpha}(f(x))=\frac{1}{\Gamma(n-\alpha)} \frac{d^{n}}{d x^{n}} \int_{0}^{x}(x-t)^{n-\alpha-1} f(t) d t, \\
n-1 \leq \alpha \leq n .
\end{array}
$$

Definition 5. The Jumarie fractional-order derivative is given as follows [16]:

$$
\begin{array}{r}
D_{x}^{\alpha}(f(x))=\frac{1}{\Gamma(n-\alpha)} \frac{d^{n}}{d x^{n}} \int_{0}^{x}(x-t)^{n-\alpha-1}\{f(t)-f(0)\} d t \\
n-1 \leq \alpha \leq n .
\end{array}
$$

Lemma 6. If $m-1<\alpha \leq m, m \in \mathbb{N}$, and $f \in C_{\mu}^{m}, \mu \geq-1$, then

$$
\begin{gathered}
D^{\alpha} J^{\alpha} f(x)=f(x), \\
J^{\alpha} D_{0}^{\alpha} f(x)=f(x)-\sum_{k=0}^{m-1} f^{(k)}\left(0^{+}\right) \frac{x^{k}}{k !}, \\
x>0 .
\end{gathered}
$$

Definition 7 (partial derivatives of fractional order $[7,8,11$, 18]). Assume now that $f(\mathbf{x})$ is a function of $n$ variables $x_{i}$, $i=1, \ldots, n$, also of class $C$ on $D \in \mathbb{R}_{n}$. We define partial derivative of order $\alpha$ for $f$ in respect to the $x_{i}$ the function as follows:

$$
a \partial_{\underline{\mathbf{x}}}^{\alpha} f=\left.\frac{1}{\Gamma(m-\alpha)} \int_{a}^{x_{i}}\left(x_{i}-t\right)^{m-\alpha-1} \partial_{x_{i}}^{m} f\left(x_{j}\right)\right|_{x_{j}=t} d t
$$

where $\partial_{x_{i}}^{m}$ is the usual partial derivative of integer order $m$.

\section{Examination of the Numerical Solution via Difference Schemes}

This section is devoted to the discussion underpinning the numerical simulation of the solution above (1) via the explicit scheme [19-23] and the implicit scheme [23-30]. However, before we present the numerical schemes, we must assume that (1) has a unique and sufficiently smooth solution [23]. In addition, to present the numerical schemes, we let $x_{l}=l h$, $0 \leq l \leq M, M h=L, t_{k}=k \tau, 0 \leq k \leq N$, and $N \tau=T ; h$ is the space step size, and $M$ and $N$ are grid points. We will start with the implicit scheme.

3.1. Implicit Scheme for the Main Problem. It is important to recall that the finite difference approximation for the secondorder spatial derivative is known as follows [23]:

$$
\begin{aligned}
\frac{\partial^{2} v\left(x_{l}, t_{k+1}\right)}{\partial x^{2}}= & \frac{v\left(x_{l+1}, t_{k+1}\right)-2 v\left(x_{l}, t_{k+1}\right)+v\left(x_{l-1}, t_{k+1}\right)}{h^{2}} \\
& +O\left(h^{2}\right) .
\end{aligned}
$$

The discretization of the Caputo-type time-fractional-order derivative can be presented as follows:

$$
\begin{aligned}
& \frac{\partial^{\alpha} v\left(x_{l}, t_{k+1}\right)}{\partial t^{\alpha}} \\
& =\frac{\tau^{-\alpha}}{\Gamma(2-\alpha)}\left(v\left(x_{l}, t_{k+1}\right)-v\left(x_{l}, t_{k}\right)\right. \\
& +\sum_{j=1}^{k}\left[v\left(x_{l}, t_{k+1-j}\right)-v\left(x_{l}, t_{k-j}\right)\right] \\
& \left.\quad \times\left[(j+1)^{1-\alpha}-(j)^{1-\alpha}\right]\right), \\
& \frac{\partial v\left(x_{l}, t_{k+1}\right)}{\partial t}=\frac{v\left(x_{l}, t_{k+1}\right)-v\left(x_{l}, t_{k}\right)}{\Delta t}+O(\Delta t)
\end{aligned}
$$

Now, substituting (9) and (10) into (1), we obtained the following expression:

$$
\begin{aligned}
& \frac{v\left(x_{l}, t_{k+1}\right)-v\left(x_{l}, t_{k}\right)}{\Delta t}+\frac{\tau^{-\alpha}}{\Gamma(2-\alpha)} \\
& \times\left(v\left(x_{l}, t_{k+1}\right)-v\left(x_{l}, t_{k}\right)\right. \\
& \quad+\sum_{j=1}^{k}\left[v\left(x_{l}, t_{k+1-j}\right)-v\left(x_{l}, t_{k-j}\right)\right]
\end{aligned}
$$




$$
\begin{gathered}
\left.\times\left[(j+1)^{1-\alpha}-(j)^{1-\alpha}\right]\right) \\
-a_{l}^{k}\left(\frac{v\left(x_{l+1}, t_{k+1}\right)-2 v\left(x_{l}, t_{k+1}\right)+v\left(x_{l-1}, t_{k+1}\right)}{h^{2}}\right) \\
+\delta v\left(x_{l}, t_{k}\right)=f\left(x_{l}, t_{k}\right) .
\end{gathered}
$$

For ease, let

$$
\begin{gathered}
v_{l}^{k}=v\left(x_{l}, t_{k}\right), \quad f_{l}^{k}=f\left(x_{l}, t_{k}\right), \\
a\left(x_{l}, t_{k}\right)=a_{l}^{k}, \quad(j+1)^{1-\alpha}-(j)^{1-\alpha}=b_{j} .
\end{gathered}
$$

Then, (11) can be rephrased as follows:

$$
\begin{aligned}
(\Delta t & \left.+\frac{\tau^{-\alpha}}{\Gamma(2-\alpha)}+2 \frac{a_{l}^{k}}{h^{2}}\right) v_{l}^{k+1} \\
= & \left(\Delta t+\frac{\tau^{-\alpha}}{\Gamma(2-\alpha)}+\delta\right) v_{l}^{k}+a_{l}^{k} v_{l+1}^{k+1} \\
& +a_{l}^{k} v_{l-1}^{k+1}-\frac{\tau^{-\alpha}}{\Gamma(2-\alpha)} \sum_{j=1}^{k}\left[v_{l}^{k-j+1}-v_{l}^{k-j}\right] b_{j}+f_{l}^{k} .
\end{aligned}
$$

It is important to inform that if $k=0$, then the term of the sum of the right-hand side automatically vanished. Then, (13) can be divided as follows:

$$
\left(\Delta t+\frac{\tau^{-\alpha}}{\Gamma(2-\alpha)}+2 \frac{a_{l}^{1}}{h^{2}}\right) v_{l}^{1}-a_{l}^{1} v_{l+1}^{1}-a_{l}^{1} v_{l-1}^{1}
$$

$$
=\left(\Delta t+\frac{\tau^{-\alpha}}{\Gamma(2-\alpha)}+\delta\right) v_{l}^{0}+f_{l}^{0}, \quad k=0,
$$

$$
\left(\Delta t+\frac{\tau^{-\alpha}}{\Gamma(2-\alpha)}+2 \frac{a_{l}^{k}}{h^{2}}\right) v_{l}^{k+1}-a_{l}^{k} v_{l+1}^{k+1}-a_{l}^{k} v_{l-1}^{k+1}
$$

$$
\begin{aligned}
= & \left(\Delta t+\frac{\tau^{-\alpha}}{\Gamma(2-\alpha)}+\delta\right) v_{l}^{k} \\
& -\frac{\tau^{-\alpha}}{\Gamma(2-\alpha)} \sum_{j=1}^{k}\left[v_{l}^{k-j}\right] d_{j}+b_{k+1} v_{j}^{0}+f_{l}^{k},
\end{aligned}
$$

where $d_{j}=b_{j+1}-b_{j}$. follows:

The above equation can be written in matrix form as

$$
\begin{aligned}
& \left(\begin{array}{cccccc}
\Delta t+\frac{\tau^{-\alpha}}{\Gamma(2-\alpha)}+2 \frac{a_{l}^{1}}{h^{2}}-a_{1}^{k} & & 0 & 0 & & \\
-a_{2}^{k} \Delta t+\frac{\tau^{-\alpha}}{\Gamma(2-\alpha)}+2 \frac{a_{2}^{1}}{h^{2}} & \ddots & 0 & 0 & 0 \\
\vdots & -a_{m-2}^{k} \Delta t+\frac{\tau^{-\alpha}}{\Gamma(2-\alpha)}+2 \frac{a_{m-2}^{1}}{h^{2}} & -a_{m-2}^{k} & 0 \\
0 & & 0 & 0-a_{m-1}^{k} & \Delta t+\frac{\tau^{-\alpha}}{\Gamma(2-\alpha)}+2 \frac{a_{l}^{1}}{h^{2}}
\end{array}\right)\left(\begin{array}{c}
v_{1}^{k+1} \\
v_{m-1}^{k+1} \\
v_{m-1}^{k+1}
\end{array}\right) \\
& =\left(\begin{array}{c}
f_{1}^{k}-\frac{a_{1}^{k}}{h^{2}} v\left(0, t_{k}\right) \\
f_{2}^{k} \\
\vdots \\
f_{m-2}^{k} \\
f_{m-1}^{k}-\frac{a_{m-1}^{k}}{h^{2}} v\left(0, t_{k}\right)
\end{array}\right) .
\end{aligned}
$$

3.1.1. Stability of the Implicit Difference Scheme. In this subsection, we present the stability analysis of the implicit difference scheme for solving the time-fractional parabolic equation (1). To accomplish this, we let $\zeta_{l}^{k}=v_{l}^{k}-V_{l}^{k}$, with $V_{l}^{k}$ being the approximate solution of the main problem at the point $\left(x_{l}, t_{k}\right), k=1, \ldots, N ; l=1, \ldots, M$; in addition, $\zeta^{k}$ is the transpose of the matrix $\left[\zeta_{1}^{k}, \zeta_{2}^{k}, \ldots, \zeta_{M}^{k}\right]$.
To analyze the stability, we exploit the Fourier method [22], and the expression of $\zeta^{k}$ can be defined as follows:

$$
\zeta^{k}(x)=\left\{\begin{array}{rc}
\zeta_{l}^{k}, & \text { if } x_{l}-\frac{h}{2}<x \leq x_{l}+\frac{h}{2}, \\
& l=1,2, \ldots, M-1, \\
0, & \text { if } L-\frac{h}{2}<x \leq L .
\end{array}\right.
$$


Then, the function $\zeta^{k}(x)$ can be expressed in Fourier series as follows:

$$
\begin{gathered}
\zeta^{k}(x)=\sum_{m=-\infty}^{m=\infty} \delta_{m}(m) \exp \left[\frac{2 i \pi m k}{L}\right], \\
\delta_{k}(x)=\frac{1}{L} \int_{0}^{L} \rho^{k}(x) \exp \left[\frac{2 i \pi m x}{L}\right] d x .
\end{gathered}
$$

It was proven in $[22,29,30]$ that

$$
\left\|\rho^{2}\right\|_{2}^{2}=\sum_{m=-\infty}^{m=\infty}\left\|\delta_{k}(m)\right\|^{2} .
$$

Let us now examine the stability of the implicit scheme of the main problem

$$
\begin{aligned}
& \left(\Delta t+\frac{\tau^{-\alpha}}{\Gamma(2-\alpha)}+2 \frac{a_{l}^{k}}{h^{2}}\right) \zeta_{l}^{k+1} \\
& =\left(\Delta t+\frac{\tau^{-\alpha}}{\Gamma(2-\alpha)}+\delta\right) \zeta_{l}^{k}+a_{l}^{k} \zeta_{l+1}^{k+1}+a_{l}^{k} \zeta_{l-1}^{k+1} \\
& \quad-\frac{\tau^{-\alpha}}{\Gamma(2-\alpha)} \sum_{j=1}^{k}\left[\zeta_{l}^{k-j}\right] d_{j}+b_{k+1} \zeta_{j}^{0}+f_{l}^{k}, \\
& k=1, \ldots, M .
\end{aligned}
$$

We next assume that

$$
\zeta_{l}^{k}=\delta_{k} \exp [i \rho l k]
$$

with $\rho$ being the real space wave number and $i=\sqrt{-1}$. Then, by replacing (21) into (20), we obtain the following:

$$
\begin{gathered}
\left(\Delta t+\frac{\tau^{-\alpha}}{\Gamma(2-\alpha)}+2 \sin ^{2}\left(\frac{\rho h}{2}\right) \frac{a_{l}^{k}}{h^{2}}\right) \delta_{k+1} \\
=\left(\Delta t+\frac{\tau^{-\alpha}}{\Gamma(2-\alpha)}+2 \sin ^{2}\left(\frac{\rho h}{2}\right) \delta-d_{1}\right) \delta_{k} \\
-\frac{\tau^{-\alpha}}{\Gamma(2-\alpha)} \sum_{j=1}^{k-1}\left[\delta_{k-j}\right] d_{j}+b_{k+1} \delta_{0}+f_{l}^{k}, \\
k=0, \ldots, M-1 .
\end{gathered}
$$

Lemma 8. Assume that $\delta_{k}(k=0, \ldots, M-1)$ verified (14) and that for all $(l, k), \delta \leq a_{l}^{k} / h^{2}+d_{1}(l=1, \ldots, N ; k=1, \ldots, M)$; then, the following inequality is satisfied:

$$
\left|\delta_{k}\right| \leq\left|\delta_{0}\right|, \quad k=1,2, \ldots, M \text {. }
$$

Proof. To prove this lemma, we make use of the recursive method on the natural number $n$ from (3) and (15), we have that, for all $l=1, \ldots, N$ and for $k=0$,

$$
\begin{aligned}
& \left(\Delta t+\frac{\tau^{-\alpha}}{\Gamma(2-\alpha)}+2 \sin ^{2}\left(\frac{\rho h}{2}\right) \frac{a_{l}^{0}}{h^{2}}\right) \delta_{1} \\
& =\left(\Delta t+\frac{\tau^{-\alpha}}{\Gamma(2-\alpha)}+2 \sin ^{2}\left(\frac{\rho h}{2}\right) \delta-d_{1}\right) \delta_{0} .
\end{aligned}
$$

And for $k \geq 1$, we have

$$
\begin{aligned}
(\Delta t & \left.+\frac{\tau^{-\alpha}}{\Gamma(2-\alpha)}+2 \sin ^{2}\left(\frac{\rho h}{2}\right) \frac{a_{l}^{k}}{h^{2}}\right) \delta_{k+1} \\
= & \left(\Delta t+\frac{\tau^{-\alpha}}{\Gamma(2-\alpha)}+2 \sin ^{2}\left(\frac{\rho h}{2}\right) \delta-d_{1}\right) \delta_{k} \\
& -\frac{\tau^{-\alpha}}{\Gamma(2-\alpha)} \sum_{j=1}^{k-1}\left[\delta_{k-j}\right] d_{j}+b_{k+1} \delta_{0} .
\end{aligned}
$$

Observe that $\delta \leq a_{l}^{0} / h^{2}+d_{1}$ then, the following is obtained:

$$
\delta_{1}=\frac{\left(\Delta t+\tau^{-\alpha} / \Gamma(2-\alpha)+2 \sin ^{2}(\rho h / 2) \delta-d_{1}\right)}{\left(\Delta t+\tau^{-\alpha} / \Gamma(2-\alpha)+2 \sin ^{2}(\rho h / v)\left(a_{l}^{0} / h^{2}\right)\right)} \delta_{0} .
$$

Applying the absolute value on both sides of (20), we have

$$
\left|\delta_{1}\right| \leq\left|\delta_{0}\right|
$$

Now, let us assume that (18) is true for all $2 \leq m \leq k$; then,

$$
\begin{aligned}
\delta_{k+1}= & \left(\left(\Delta t+\frac{\tau^{-\alpha}}{\Gamma(2-\alpha)}+2 \sin ^{2}\left(\frac{\rho h}{2}\right) \delta-d_{1}\right) \delta_{k}\right. \\
& \left.-\frac{\tau^{-\alpha}}{\Gamma(2-\alpha)} \sum_{j=1}^{k-1}\left[\delta_{k-j}\right] d_{j}+b_{k+1} \delta_{0}\right) \\
& \times\left(\Delta t+\frac{\tau^{-\alpha}}{\Gamma(2-\alpha)}+2 \sin ^{2}\left(\frac{\rho h}{2}\right) \frac{a_{l}^{k}}{h^{2}}\right)^{-1} .
\end{aligned}
$$

Now, applying the absolute value on both sides of (28), and making further use of the inequality theorem, we arrived at the following:

$$
\begin{aligned}
\left|\delta_{k+1}\right| \leq & \left(\left|\Delta t+\frac{\tau^{-\alpha}}{\Gamma(2-\alpha)}+2 \sin ^{2}\left(\frac{\rho h}{2}\right) \delta-d_{1}\right|\left|\delta_{k}\right|\right. \\
& \left.+\frac{\tau^{-\alpha}}{\Gamma(2-\alpha)} \sum_{j=1}^{k-1}\left|\delta_{k-j}\right| d_{j}+b_{k+1}\left|\delta_{0}\right|\right) \\
& \times\left(\left|\Delta t+\frac{\tau^{-\alpha}}{\Gamma(2-\alpha)}+2 \sin ^{2}\left(\frac{\rho h}{2}\right) \frac{a_{l}^{k}}{h^{2}}\right|\right)^{-1} .
\end{aligned}
$$

Making use of the induction hypothesis and factorizing $\left|\delta_{0}\right|$, we obtain

$$
\begin{aligned}
\left|\delta_{k+1}\right| \leq[(\Delta t & +\frac{\tau^{-\alpha}}{\Gamma(2-\alpha)}+2 \sin ^{2}\left(\frac{\rho h}{2}\right) \delta-d_{1} \\
& \left.+\frac{\tau^{-\alpha}}{\Gamma(2-\alpha)} \sum_{j=1}^{k-1} d_{j}+b_{k+1}\right) \\
& \left.\times\left(\left|\Delta t+\frac{\tau^{-\alpha}}{\Gamma(2-\alpha)}+2 \sin ^{2}\left(\frac{\rho h}{2}\right) \frac{a_{l}^{k}}{h^{2}}\right|\right)^{-1}\right]\left|\delta_{0}\right| .
\end{aligned}
$$


But we have that

$$
\sum_{j=1}^{k-1} d_{j}=1-b_{k+1}, \quad 0 \leq d_{j} \leq 1
$$

Therefore,

$$
\left|\delta_{k+1}\right| \leq\left[\frac{\Delta t+\tau^{-\alpha} / \Gamma(2-\alpha)+2 \sin ^{2}(\rho h / 2) \delta-d_{1}}{\left|\Delta t+\tau^{-\alpha} / \Gamma(2-\alpha)+2 \sin ^{2}(\rho h / 2)\left(a_{l}^{k} / h^{2}\right)\right|}\right]\left|\delta_{0}\right| .
$$

Thus,

$$
\left|\delta_{k+1}\right| \leq\left|\delta_{0}\right|
$$

Theorem 9. The implicit difference scheme for the timefractional parabolic equation (1) is stable providing that, for all $(l, k), \delta \leq a_{l}^{k} / h^{2}+d_{1}(l=1, \ldots, N ; k=1, \ldots, M)$.

Proof. From (19) and Lemma 8, we obtain

$$
\left\|\zeta^{2}\right\|_{2} \leq\left\|\zeta^{0}\right\|_{2}
$$

and this proves that the implicit difference scheme for the time-fractional parabolic equation (1) is stable.

Remark 10. It is observed that, from Theorem 9, the statement of stability of implicit difference scheme for time-fractional parabolic equation (1) depends on the evolution of the function $a(x, t)$. It follows that the stability condition can change in time advancement and space position.

3.1.2. Convergence Analysis of the Implicit Difference Scheme. Assuming that $v\left(x_{l}, t_{k}\right)(l=0, \ldots, M-1 ; k=0, \ldots, N-1)$ is the exact solution of (1) at the point $\left(x_{l}, t_{k}\right)$, then, by defining, $\beta_{l}^{k}=v\left(x_{l}, t_{k}\right)-v_{l}^{k}$, and $\beta^{k}$ is the transpose of the matrix $\left(\beta_{1}^{k}, \beta_{2}^{k}, \ldots, \beta_{N}^{k}\right)$; here, $\beta^{0}$ is neglected because of being equal to zero. Therefore, we have the following relation for the implicit difference scheme for the time-fractional parabolic equation (1) and from (13):

$$
\begin{aligned}
(\Delta t & \left.+\frac{\tau^{-\alpha}}{\Gamma(2-\alpha)}+2 \frac{a_{l}^{0}}{h^{2}}\right) \beta_{l}^{1}-a_{l}^{0} \beta_{l+1}^{1} \\
& -a_{l}^{0} \beta_{l-1}^{1}-f_{l}^{0}=T_{l}^{1}, \quad \text { for } k=0, \\
(\Delta t & \left.+\frac{\tau^{-\alpha}}{\Gamma(2-\alpha)}+2 \frac{a_{l}^{k}}{h^{2}}\right) \beta_{l}^{k+1}-\left(\Delta t+\frac{\tau^{-\alpha}}{\Gamma(2-\alpha)}+\delta\right) \beta_{l}^{k} \\
& -a_{l}^{k} \beta_{l+1}^{k+1}-a_{l}^{k} \beta_{l-1}^{k+1}-f_{l}^{k} \\
= & -\frac{\tau^{-\alpha}}{\Gamma(2-\alpha)} \sum_{j=1}^{k-1} \beta_{l}^{k-j} b_{j}+T_{l}^{k}, \quad \text { for } k \geq 1 .
\end{aligned}
$$

Here, $T_{l}^{k+1}$ is the truncate or the remainder term of the approximation and has the following expression:

$$
\begin{aligned}
T_{l}^{k+1}= & v\left(x_{l}, t_{k+1}\right)+\frac{\tau^{-\alpha}}{\Gamma(2-\alpha)} \sum_{j=1}^{k-1} \beta_{l}^{k-j} b_{j} \\
& +a\left(x_{l}, t_{k}\right)\left[v\left(x_{l+1}, t_{k+1}\right)\right. \\
& \left.-2 v\left(x_{l}, t_{k+1}\right)+v\left(x_{l-1}, t_{k+1}\right)\right] \\
+ & \left(v\left(x_{l}, t_{k+1}\right)-v\left(x_{l}, t_{k}\right)\right)-f\left(x_{l}, t_{k}\right)+\delta v\left(x_{l}, t_{k}\right) .
\end{aligned}
$$

It follows from (3), (4), and (5) that,

$$
\begin{aligned}
& \frac{\partial^{\alpha} v\left(x_{l}, t_{k+1}\right)}{\partial t^{\alpha}}+D_{1} \tau \\
& =\frac{\tau^{-\alpha}}{\Gamma(2-\alpha)} \\
& \quad \times\left(v\left(x_{l}, t_{k+1}\right)-v\left(x_{l}, t_{k}\right)\right. \\
& \left.\quad+\sum_{j=1}^{k-1} v\left(x_{l}, t_{k-j}\right) d_{j}+d_{k+1} v\left(x_{l}, t_{0}\right)\right), \\
& \frac{\partial^{2} v\left(x_{l}, t_{k+1}\right)}{\partial x^{2}}+h^{2} D_{2} \\
& =\frac{v\left(x_{l+1}, t_{k+1}\right)-2 v\left(x_{l}, t_{k+1}\right)+v\left(x_{l-1}, t_{k+1}\right)}{h^{2}}, \\
& \frac{\partial v\left(x_{l}, t_{k+1}\right)}{\partial t}+\Delta t D_{3}=\frac{v\left(x_{l}, t_{k+1}\right)-v\left(x_{l}, t_{k}\right)}{\Delta t} .
\end{aligned}
$$

Thus, from (36) and (37), we have the following:

$$
T_{l}^{k+1} \leq D\left(\tau^{1+\alpha}+h^{2} \tau^{\alpha}+\Delta t \tau^{\alpha}\right)
$$

where $D_{1}, D_{2}, D_{3}$, and $D$ are constant. The interested reader can find the error analysis of the chosen fractional derivative (the Caputo fractional derivative) in $[29,30]$.

Lemma 11. One has that $\left\|\beta^{k}\right\|_{\infty} \leq D\left(b^{k+1}\right)^{-1}\left[\tau^{1+\alpha}+\tau^{\alpha} h^{2}+\right.$ $\left.\Delta t \tau^{\alpha}\right]$ is true for all $k=0,1, \ldots, M-1$.

Proof. Again we employ the induction method to achieve this, so that, for $k=0$,

$$
\begin{gathered}
\left(\Delta t+\frac{\tau^{-\alpha}}{\Gamma(2-\alpha)}+2 \frac{a_{l}^{0}}{h^{2}}\right)\left|\beta_{l}^{1}\right|-a_{l}^{0}\left|\beta_{l+1}^{1}\right|-a_{l}^{0}\left|\beta_{l-1}^{1}\right| \geq\left|\beta_{l}^{1}\right| \\
\left|T_{l}^{1}\right|=\left|\left(\Delta t+\frac{\tau^{-\alpha}}{\Gamma(2-\alpha)}+2 \frac{a_{l}^{0}}{h^{2}}\right) \beta_{l}^{1}-a_{l}^{0} \beta_{l+1}^{1}-a_{l}^{0} \beta_{l-1}^{1}\right| \\
\geq\left|\beta_{l}^{1}\right| \\
D\left(b^{1}\right)^{-1}\left(\tau^{1+\alpha}+h^{2} \tau^{\alpha}+\Delta t \tau^{\alpha}\right) \geq\left|T_{l}^{1}\right|
\end{gathered}
$$


Now, assuming that, for all $j=0, \ldots, M-2,\left\|\beta^{j}\right\|_{\infty} \leq$ $D\left(b^{j+1}\right)^{-1}\left[\tau^{1+\alpha}+\tau^{\alpha} h^{2}+\Delta t \tau^{\alpha}\right]$, then,

$$
\begin{aligned}
& \left(\Delta t+\frac{\tau^{-\alpha}}{\Gamma(2-\alpha)}+2 \frac{a_{l}^{k}}{h^{2}}\right)\left|\beta_{l}^{k+1}\right| \\
& \quad-\left(\Delta t+\frac{\tau^{-\alpha}}{\Gamma(2-\alpha)}+\delta\right)\left|\beta_{l}^{k}\right|-a_{l}^{k}\left|\beta_{l+1}^{k+1}\right|-a_{l}^{k}\left|\beta_{l-1}^{k+1}\right| \\
& \geq\left|\beta_{l}^{k+1}\right|, \\
& \mid\left(\Delta t+\frac{\tau^{-\alpha}}{\Gamma(2-\alpha)}+2 \frac{a_{l}^{k}}{h^{2}}\right) \beta_{l}^{k+1} \\
& \quad-\left(\Delta t+\frac{\tau^{-\alpha}}{\Gamma(2-\alpha)}+\delta\right) \beta_{l}^{k}-a_{l}^{k} \beta_{l+1}^{k+1}-a_{l}^{k} \beta_{l-1}^{k+1} \mid \\
& \geq\left|\beta_{l}^{k+1}\right|, \\
& \left|\frac{\tau^{-\alpha}}{\Gamma(2-\alpha)} \sum_{j=1}^{k-1} \beta_{l}^{k-j} b_{j}+T_{l}^{k+1}\right| \geq\left|\beta_{l}^{k+1}\right|, \\
& \quad \frac{\tau^{-\alpha} \sum_{j=1}^{k-1}\left|\beta_{l}^{k-j}\right| b_{j}+T_{l}^{k+1} \geq\left|\beta_{l}^{k+1}\right| .}{\Gamma(2-\alpha)}
\end{aligned}
$$

Making use of the induction hypothesis, we obtain the following:

$$
\begin{gathered}
\frac{\tau^{-\alpha}}{\Gamma(2-\alpha)} \sum_{j=1}^{k-1}\left\|\beta_{l}^{k-j}\right\|_{\infty} b_{j}+\left[\tau^{1+\alpha}+\tau^{\alpha} h^{2}+\Delta t \tau^{\alpha}\right] \geq\left|\beta_{l}^{k+1}\right| \\
\left(b^{0}+b^{k+1}-b^{k+1}\right)\left(b^{k+1}\right)^{-1} \times D\left[\tau^{1+\alpha}+\tau^{\alpha} h^{2}+\Delta t \tau^{\alpha}\right] \\
\geq\left|\beta_{l}^{k+1}\right|, \\
\left(b^{k+1}\right)^{-1} \times D\left[\tau^{1+\alpha}+\tau^{\alpha} h^{2}+\Delta t \tau^{\alpha}\right] \geq\left|\beta_{l}^{k+1}\right| .
\end{gathered}
$$

This completes the proof.

Theorem 12. The implicit difference scheme of the timefractional parabolic equation (1) is convergent, and there exists a constant D such that,

$$
\begin{aligned}
& \left|v\left(x_{l}, t_{k}\right)-v_{l}^{k}\right| \leq D\left(\tau^{1+\alpha}+\tau^{\alpha} h^{2}+\Delta t \tau^{\alpha}\right), \\
& \quad \text { for }(l=0, \ldots, N-1 ; k=0, \ldots, M-1) .
\end{aligned}
$$

3.2. Explicit Difference Scheme for Time-Fractional Parabolic Equation. It is important to recall that the finite-difference approximation for the second-order spatial derivative is known as follows:

$$
\frac{\partial^{2} v\left(x_{l}, t_{k}\right)}{\partial x^{2}}=\frac{v\left(x_{l+1}, t_{k}\right)-2 v\left(x_{l}, t_{k}\right)+v\left(x_{l-1}, t_{k}\right)}{h^{2}}+O\left(h^{2}\right) \text {. }
$$

The discretization of the Caputo-type time-fractional-order derivative can be presented as follows:

$$
\begin{aligned}
& \frac{\partial^{\alpha} v\left(x_{l}, t_{k+1}\right)}{\partial t^{\alpha}} \\
& =\frac{\tau^{-\alpha}}{\Gamma(2-\alpha)}\left(v\left(x_{l}, t_{k+1}\right)-v\left(x_{l}, t_{k}\right)\right. \\
& +\sum_{j=1}^{k}\left[v\left(x_{l}, t_{k+1-j}\right)-v\left(x_{l}, t_{k-j}\right)\right] \\
& \left.\quad \times\left[(j+1)^{1-\alpha}-(j)^{1-\alpha}\right]\right), \\
& \frac{\partial v\left(x_{l}, t_{k}\right)}{\partial t}=\frac{v\left(x_{l+1}, t_{k}\right)-v\left(x_{l}, t_{k}\right)}{\Delta t}+O(\Delta t) .
\end{aligned}
$$

Now, substituting (44) and (45) into (1), we obtained the following expression:

$$
\begin{aligned}
& \frac{v\left(x_{l+1}, t_{k}\right)-v\left(x_{l}, t_{k}\right)}{\Delta t} \\
& +\frac{\tau^{-\alpha}}{\Gamma(2-\alpha)}\left(v\left(x_{l}, t_{k+1}\right)-v\left(x_{l}, t_{k}\right)\right. \\
& +\sum_{j=1}^{k}\left[v\left(x_{l}, t_{k+1-j}\right)-v\left(x_{l}, t_{k-j}\right)\right] \\
& \left.\times\left[(j+1)^{1-\alpha}-(j)^{1-\alpha}\right]\right) \\
& -a_{l}^{k}\left(\frac{v\left(x_{l+1}, t_{k}\right)-2 v\left(x_{l}, t_{k}\right)+v\left(x_{l-1}, t_{k}\right)}{h^{2}}\right) \\
& +\delta v\left(x_{l}, t_{k}\right)=f\left(x_{l}, t_{k}\right) .
\end{aligned}
$$

For simplicity, let

$$
\begin{gathered}
v_{l}^{k}=v\left(x_{l}, t_{k}\right), \quad f_{l}^{k}=f\left(x_{l}, t_{k}\right), \\
a\left(x_{l}, t_{k}\right)=a_{l}^{k}, \quad(j+1)^{1-\alpha}-(j)^{1-\alpha}=b_{j} .
\end{gathered}
$$

Then, (37) can be rewritten as follows

$$
\begin{aligned}
v_{l}^{k+1}= & v_{l}^{k}\left[B_{1}+1-r_{l}^{k}-\delta\right]+v_{l+1}^{k}\left[r_{l}^{k}-B_{1}\right] \\
& +r_{l}^{k} v_{l-1}^{k}+B_{1} f_{l}^{k}-\sum_{j=1}^{k}\left[v_{l}^{k+1-j}-v_{l}^{k-j}\right] b_{j},
\end{aligned}
$$

where $B_{1}=\Gamma(2-\alpha) \tau^{\alpha}$ and $r_{l}^{k}=\left(\Gamma(2-\alpha) \tau^{\alpha} / h^{2}\right) a_{l}^{k}$.

3.2.1. Stability of the Explicit Difference Scheme of the TimeFractional Parabolic Equation. Following the discussion presented earlier for the analysis of the implicit scheme, we 
obtain the following roundoff error equation from explicit scheme equation (1):

$$
\begin{aligned}
\beta_{l}^{k+1}=\beta_{l}^{k}\left[B_{1}+1-r_{l}^{k}-\delta\right]+\beta_{l+1}^{k}\left[r_{l}^{k}-B_{1}\right] \\
+r_{l}^{k} \beta_{l-1}^{k}+B_{1} f_{l}^{k}-\sum_{j=1}^{k}\left[\beta_{l}^{k-j}\right] d_{j}-d_{k+1} \beta^{0} \\
\\
\text { for } k=1, \ldots, M-1,
\end{aligned}
$$

where

$$
d_{j}=b_{j+1}-b_{j}
$$

Again, we suppose that $\beta_{l}^{k}$ in (39) can be given in exponential form as follows:

$$
\beta_{l}^{k}=\delta_{k} e^{(-i \sigma l k)}
$$

Then, replacing this form into (49), we arrive at the following expression:

$$
\begin{aligned}
\delta_{k+1}= & \delta_{k}\left[B_{1}+1-\sin ^{2}\left(\frac{\sigma h}{2}\right) r_{l}^{k}-\delta\right] \\
& -\sum_{j=1}^{k-1} \delta_{k-j} d_{j}+\delta_{0} d_{k+1}, \quad \text { for } k=0, \ldots, M-1 .
\end{aligned}
$$

Lemma 13. Suppose that $\delta_{k}$ is the solution of (40) for all $(l=$ $0, \ldots, N-1 ; k=0, \ldots, M-1), r_{l}^{k} \leq 1-\delta+B_{1}$, and $r_{l}^{k} \geq B_{1}-\delta$, then, the following inequality holds:

$$
\left|\delta_{k}\right| \leq\left|\delta_{0}\right|, \quad \text { for } k \geq 1 \text {. }
$$

Proof. To prove this, we make use of the recurrence technique on the natural number $k$, and we will examine first the case for $k=1$. Therefore, if $k=1$, we have that

$$
\delta_{1}=\delta_{0}\left[B_{1}+1-\sin ^{2}\left(\frac{\sigma h}{2}\right) r_{l}^{0}-\delta\right] \text {. }
$$

Since $r_{l}^{0} \leq 1-\delta+B_{1}$ and $r_{l}^{0} \geq B_{1}-\delta$, then, we have

$$
\left|\delta_{1}\right| \leq\left|\delta_{0}\right| \text {. }
$$

We next assume that, for all $j=1, \ldots, k,\left|\delta_{k}\right| \leq\left|\delta_{0}\right|$; then

$$
\begin{aligned}
\left|\delta_{k+1}\right|=\mid \delta_{k} & {\left[B_{1}+1-\sin ^{2}\left(\frac{\sigma h}{2}\right) r_{l}^{k}-\delta\right] } \\
& -\sum_{j=1}^{k-1} \delta_{k-j} d_{j}+\delta_{0} d_{k+1} \mid .
\end{aligned}
$$

Now, making use of the triangular inequality, we arrive at the following inequality:

$$
\begin{aligned}
\left|\delta_{k+1}\right| \leq & \left|\delta_{k}\left[B_{1}+1-\sin ^{2}\left(\frac{\sigma h}{2}\right) r_{l}^{k}-\delta\right]\right| \\
& +\sum_{j=1}^{k-1}\left|\delta_{k-j}\right| d_{j}+\left|\delta_{0}\right| d_{k+1} .
\end{aligned}
$$

Now, making use of the induction hypothesis, we arrive at the following:

$$
\left|\delta_{k+1}\right| \leq\left|\left[B_{1}+1-\sin ^{2}\left(\frac{\sigma h}{2}\right) r_{l}^{k}-\delta\right]+\sum_{j=1}^{k-1} d_{j}+d_{k+1}\right|\left|\delta_{0}\right| .
$$

And this produces, since $\sum_{j=1}^{k-1} d_{j}+d_{k+1}=1$,

$$
\left|\delta_{k+1}\right| \leq\left|\left[B_{1}+1-\sin ^{2}\left(\frac{\sigma h}{2}\right) r_{l}^{k}-\delta\right]\right|\left|\delta_{0}\right| \leq\left|\delta_{0}\right| .
$$

This completes the proof.

Theorem 14. The explicit difference scheme of time-fractional parabolic equation (1) is stable under the condition that, for all $(l=0,1, \ldots, N-1 ; k=0, \ldots, M-1), r_{l}^{k} \leq 1-\delta+B_{1}$ and $r_{l}^{k} \geq B_{1}-\delta$.

Proof. It is straightforward from (11) and Lemma 13, that,

$$
\left\|\zeta^{2}\right\|_{2} \leq\left\|\zeta^{0}\right\|_{2}
$$

and this proves that the explicit difference scheme for the time-fractional parabolic equation (3) is stable.

\subsubsection{Convergence Analysis of the Explicit Scheme}

Theorem 15. The explicit scheme is convergent if, for all $(l=$ $0,1, \ldots, N-1 ; k=0, \ldots, M-1), r_{l}^{k} \leq 1-\delta+B_{1}$ and $r_{l}^{k} \geq B_{1}-\delta$ and there exists a constant $D$ such that

$$
\left|v_{l}^{k}-v\left(x_{l}, t_{k}\right)\right| \leq D\left(\tau+h^{2}+\Delta t\right)
$$

Using the same method in implicit difference, the above theorem can be proven.

\section{Conclusions}

Partial differential equations are sometimes very difficult to be solved analytically. It is, therefore, sometimes easy to solve them via numerical techniques. In this paper, we solve the time-fractional parabolic equation (1) via the implicit and explicit difference schemes. We study the stability and the convergence of implicit and explicit difference schemes.

\section{References}

[1] A. Ashyralyev, F. Dal, and Z. Pinar, "On the numerical solution of fractional hyperbolic partial differential equations," Mathematical Problems in Engineering, vol. 2009, Article ID 730465, 2009.

[2] A. Ashyralyev, "A note on fractional derivatives and fractional powers of operators," Journal of Mathematical Analysis and Applications, vol. 357, no. 1, pp. 232-236, 2009.

[3] I. Podlubny and A. M. A. El-Sayed, On Two Definitions of Fractional Calculus, Slovak Academy of Science, Institute of Experimental Physics, 1996. 
[4] A. Yakar and M. E. Koksal, "Existence results for solutions of nonlinear fractional differential equations," Abstract and Applied Analysis, vol. 2012, Article ID 267108, 12 pages, 2012.

[5] A. Atangana and J. F. Botha, "Generalized groundwater flow equation using the concept of variable order derivative," Boundary Value Problems, vol. 2013, article 53, 2013.

[6] A. Atangana and S. C. Oukouomi Noutchie, "Stability and convergence of a tme-fractional variable order Hantush equation for a deformable aquifer," Abstract and Applied Analysis, vol. 2013, Article ID 691060, 8 pages, 2013.

[7] D. Baleanu, K. Diethelm, E. Scalas, and J. J. Trujillo, Fractional Calculus Models and Numerical Methods Series on Complexity, Nonlinearity and Chaos, World Scientific, 2012.

[8] A. A. Kilbas, H. M. Srivastava, and J. J. Trujillo, Theory and Applications of Fractional Differential Equations, vol. 204 of North-Holland Mathematics Studies, Elsevier Science B.V., Amsterdam, The Netherlands, 2006.

[9] K. B. Oldham and J. Spanier, The Fractional Calculus, vol. 111 of Mathematics in Science and Engineering, Academic Press, New York, NY, USA, 1974.

[10] B. Ross, Ed., Fractional Calculus and Its Applications, vol. 457 of Lecture Notes in Mathematics, Springer, Berlin, Germany, 1975.

[11] Y. Luchko and R. Gorenflo, "The initial value problem for some fractional differential equations with the Caputo derivative," Preprint Series A08-98, Freic Universitat Berlin, 1998, Fachbreich Mathematik and Informatik.

[12] A. Atangana and E. Alabaraoye, "Solving system of fractional partial differential equations arisen in the model of HIV infection of $\mathrm{CD}^{+}$cells and attractor one-dimensional KellerSegel equation," Advances in Difference Equations, vol. 2013, article 94, 2013.

[13] I. Podlubny, Fractional Differential Equations, vol. 198 of Mathematics in Science and Engineering, Academic Press, San Diego, Calif, USA, 1999.

[14] A. Atangana and A. Secer, "A Note on fractional order derivatives and table of fractional derivatives of some special functions," Abstract and Applied Analysis, vol. 2013, Article ID 279681, 8 pages, 2013.

[15] K. S. Miller and B. Ross, An Introduction to the Fractional Calculus and Fractional Differential Equations, John Wiley \& Sons, New York, NY, USA, 1993.

[16] A. Atangana and A. Kilicman, "Analytical solutions of the spacetime fractional derivative of advection dispersion equation," Mathematical Problems in Engineering, vol. 2013, Article ID 853127, 9 pages, 2013.

[17] X.-J. Yang, D. Baleanu, and J. A. Tenreiro Machado, "Systems of navier-stokes equations on cantor sets," Mathematical Problems in Engineering, vol. 2013, Article ID 769724, 8 pages, 2013.

[18] F. Mainardi, "Fractional calculus: some basic problems in continuum and statistical mechanics," in Fractals and Fractional Calculus in Continuum Mechanics, vol. 378 of CISM Courses and Lectures, pp. 291-348, Springer, Vienna, Austria, 1997.

[19] M. M. Meerschaert and C. Tadjeran, "Finite difference approximations for fractional advection-dispersion flow equations," Journal of Computational and Applied Mathematics, vol. 172, no. 1, pp. 65-77, 2004.

[20] Y. Zhang, "A finite difference method for fractional partial differential equation," Applied Mathematics and Computation, vol. 215, no. 2, pp. 524-529, 2009.

[21] C. Tadjeran, M. M. Meerschaert, and H. P. Scheffler, "A secondorder accurate numerical approximation for the fractional diffusion equation," Journal of Computational Physics, vol. 213, no. 1, pp. 205-213, 2006.

[22] C. M. Chen, F. Liu, I. Turner, and V. Anh, "A Fourier method for the fractional diffusion equation describing sub-diffusion," Journal of Computational Physics, vol. 227, no. 2, pp. 886-897, 2007.

[23] S. B. Yuste and L. Acedo, "An explicit finite difference method and a new von Neumann-type stability analysis for fractional diffusion equations," SIAM Journal on Numerical Analysis, vol. 42, no. 5, pp. 1862-1874, 2005.

[24] I. Podlubny, A. Chechkin, T. Skovranek, Y. Chen, and B. M. Vinagre Jara, "Matrix approach to discrete fractional calculus II: partial fractional differential equations," Journal of Computational Physics, vol. 228, no. 8, pp. 3137-3153, 2009.

[25] E. Hanert, "On the numerical solution of space-time fractional diffusion models," Computers and Fluids, vol. 46, no. 1, pp. 3339, 2011.

[26] P. Zhuang, F. Liu, V. Anh, and I. Turner, "Numerical methods for the variable-order fractional advection-diffusion equation with a nonlinear source term," SIAM Journal on Numerical Analysis, vol. 47, no. 3, pp. 1760-1781, 2009.

[27] R. Lin, F. Liu, V. Anh, and I. Turner, "Stability and convergence of a new explicit finite-difference approximation for the variable-order nonlinear fractional diffusion equation," Applied Mathematics and Computation, vol. 212, no. 2, pp. 435-445, 2009.

[28] J. Crank and P. Nicolson, "A practical method for numerical evaluation of solutions of partial differential equations of the heat conduction type," Proceedings of the Cambridge Philosophical Society, vol. 43, no. 1, pp. 50-67, 1947.

[29] K. Diethelm, N. J. Ford, and A. D. Freed, "Detailed error analysis for a fractional Adams method," Numerical Algorithms, vol. 36, no. 1, pp. 31-52, 2004.

[30] C. P. Li and C. X. Tao, "On the fractional Adams method," Computers and Mathematics with Applications, vol. 58, no. 8, pp. 1573-1588, 2009. 


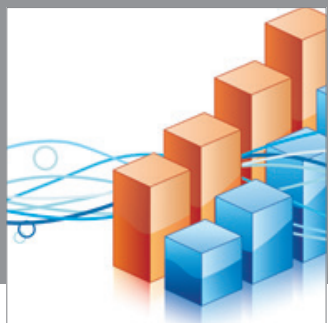

Advances in

Operations Research

mansans

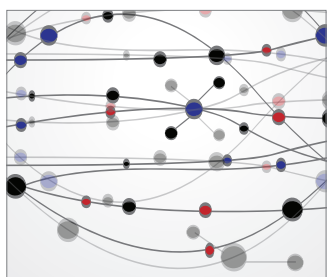

The Scientific World Journal
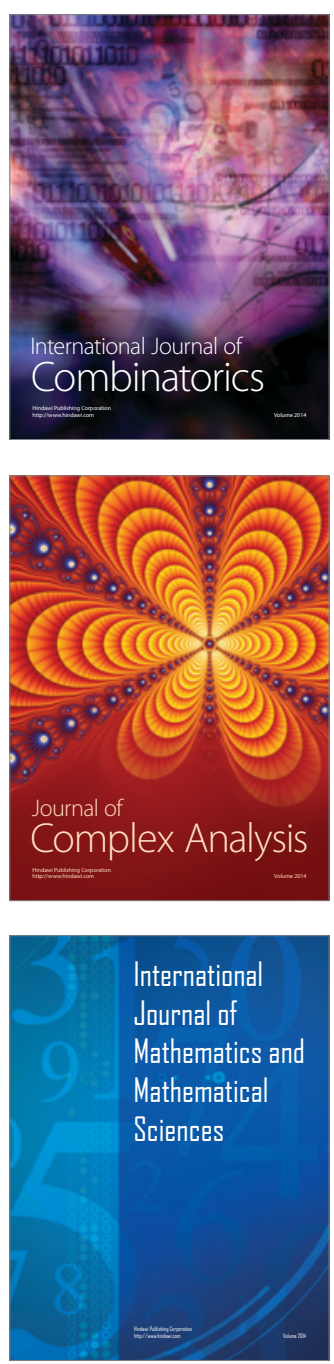
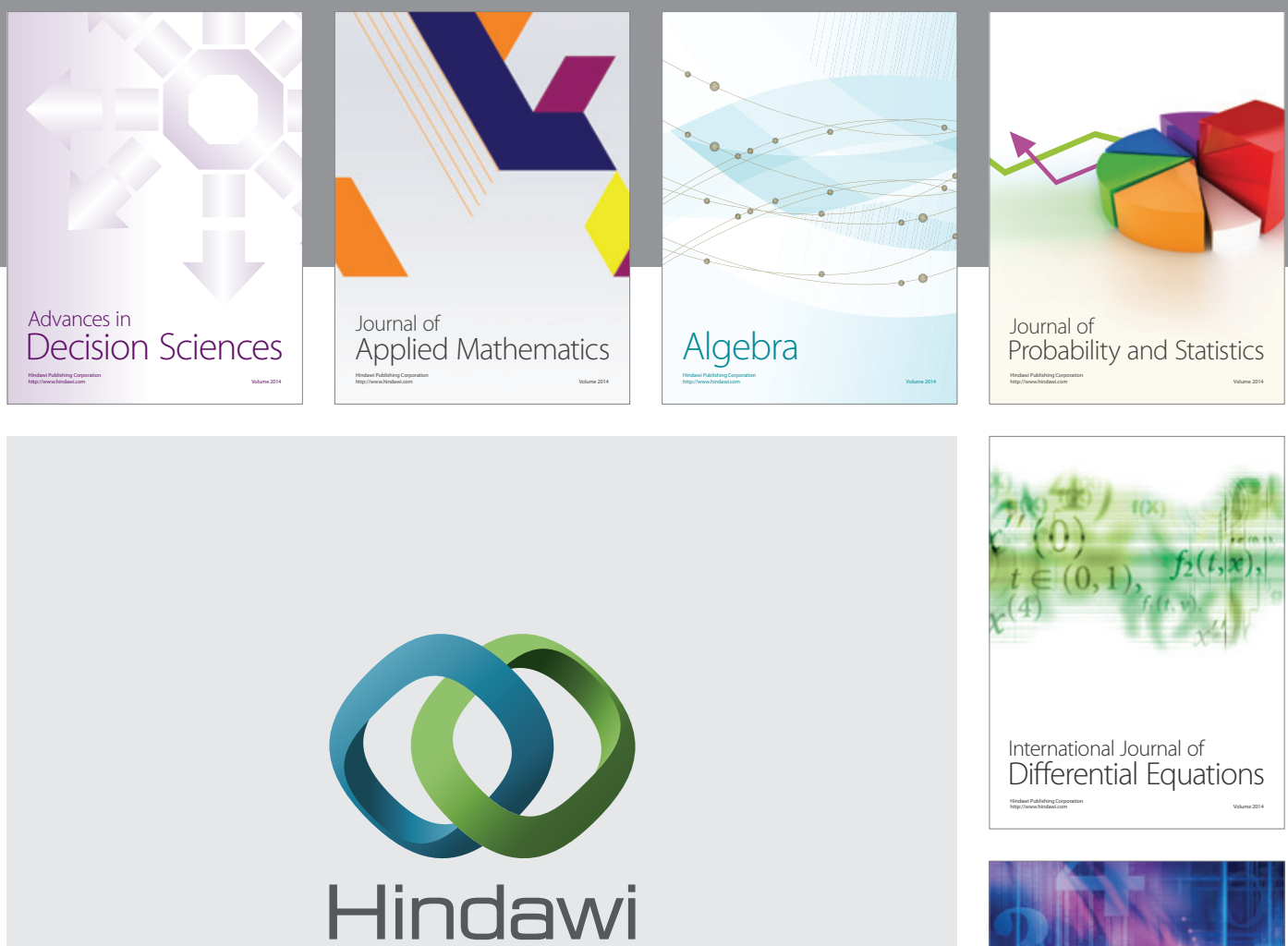

Submit your manuscripts at http://www.hindawi.com
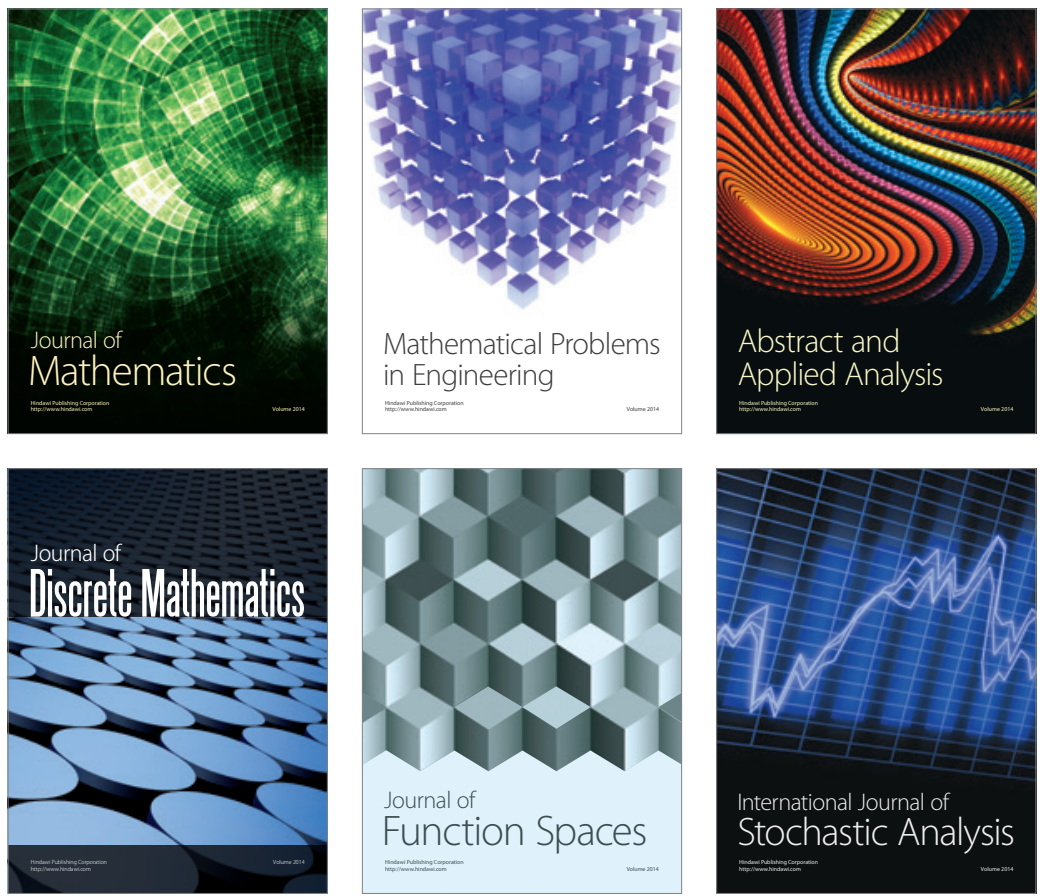

Journal of

Function Spaces

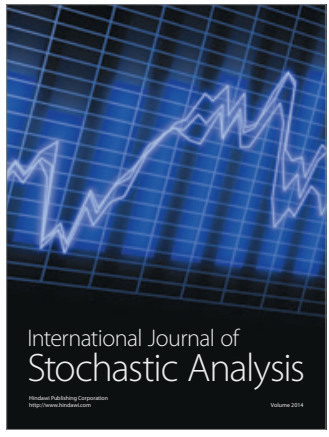

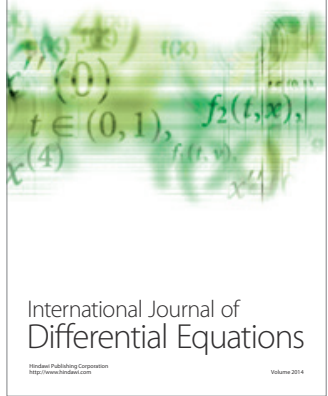
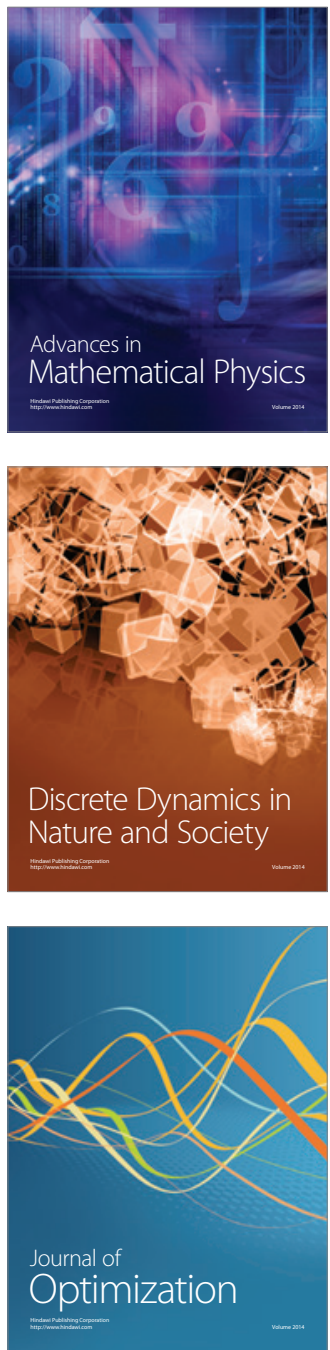